\title{
3D-printed multi-armed rotator mechanism for simultaneously testing a variety of lure blends
}

\author{
T.E.S. Sullivan, F. Mas, T.J. Welsh and D.M. Suckling \\ The New Zealand Institute for Plant \& Food Research Limited, Private Bag 4704, Christchurch 8140, \\ New Zealand \\ Corresponding author: thomas.sullivan@plantandfood.co.nz
}

With improvements in 3D-printing technology, the ability to convert the idea of an object quickly into an actual 3D object is becoming both more practicable and affordable. We have been developing a $3 \mathrm{D}$-printed gear housing and a detachable sleeve mechanism with a variable number of rotating arms, to facilitate the testing of a variety of fruit fly lures while controlling for environmental bias. The speed of the rotator mechanism is controlled using an adjustable DC step-down module and a series of 3D printed gears. Our current prototype runs on four AA batteries delivering 6V. For testing over longer timeframes or in remote areas, just about any power supply outputting between 5 and 40 volts can be attached. The model is small and has optional printed components that make the unit rain proof so it can be used for both laboratory and field trials. The model can be printed on any 3D printer with a large enough stage. This model can be used to test lure blends for a variety of insects such as flies or beetles, in a variety of settings such as laboratories, pastures, orchards and forests.

\section{A field study of Bactericera cockerelli feeding timing in potato crops}

\author{
J.N. Furlong, J. Vereijssen, A.R. Pitman, and R.C. Butler \\ The New Zealand Institute for Plant \& Food Research Limited, Private Bag 4704, Christchurch 8140, \\ New Zealand \\ Corresponding author: jess.furlong@plantandfood.co.nz
}

Since its 2006 detection in New Zealand, the tomato potato psyllid (TPP), Bactericera cockerelli, has been responsible for yield and quality-reducing damage to potatoes as well as to other solanaceous crops. TPP is best known as the insect vector for the zebra chip disease-associated bacterium Candidatus Liberibacter solanacearum (CLso), but feeding by CLso-negative TPP on potatoes is also linked to damaging effects. An improved understanding of which potato plant physiological stages are most affected by feeding of CLso-negative TPP will allow for more directed integrated pest management (IPM) plans. As part of a two-year repeated field study to assess the effect of TPP feeding timing on potatoes, CLso-negative TPP were released into mesh cages over outdoor potato ('Moonlight') plantings at five different growth stages: post-emergence, flowering, post-flowering, late-season, and pre-harvest. Tubers were grouped by plant at harvest. (Un)marketable numbers and weights, marketable dry matter and zebra chip severity were determined. Results from this trial and other shadehouse trials conducted previously are still being analysed but have the potential to better target IPM plans and reduce costly, labour-intensive, and environmentally impactful pesticide spray use, which is currently relied on heavily throughout growing seasons. 